\title{
Nestin+ Mesenchymal Precursors Generate Distinct Spleen Stromal Cell Subsets and Have Immunomodulatory Function
}

\author{
Jing Huang \\ Sun Yat-Sen University \\ Minrong Li \\ Sun Yat-Sen University \\ Ronghai Deng \\ Sun Yat-sen University First Affiliated Hospital \\ Weiqiang Li \\ Sun Yat-Sen University \\ Meihua Jiang \\ Sun Yat-Sen University \\ Andy Peng Xiang \\ Sun Yat-Sen University \\ Xiaoran Zhang ( $\nabla$ zhangxr53@mail.sysu.edu.cn ) \\ Sun Yat-Sen University https://orcid.org/0000-0001-6421-0355
}

\section{Research Article}

Keywords: Mesenchymal stromal cells, Spleen, Nestin, Immunoregulation, Inflammatory Bowel Diseases

Posted Date: November 30th, 2021

DOI: https://doi.org/10.21203/rs.3.rs-1084782/v1

License: (9) This work is licensed under a Creative Commons Attribution 4.0 International License.

Read Full License 


\section{Abstract \\ Background}

Mesenchymal stromal cells (MSCs) are known to be widespread in many tissues and possess a broad spectrum of immunoregulatory properties. They have been used in the treatment of a variety of inflammatory diseases; however, the therapeutic effects are still inconsistent owing to their heterogeneity. Spleen stromal cells have evolved to regulate the immune response at many levels as they are bathed in a complex inflammatory milieu during infection. Therefore, it is unknown whether they have stronger immunomodulatory effects than their counterparts derived from other tissues.

\section{Methods}

Here, using a transgenic mouse model expressing GFP driven by the Nestin (Nes) promoter, Nes-GFP+ cells from bone marrow and spleen were collected. Artificial lymphoid reconstruction in vivo was performed. Cell phenotype, inhibition of T cell inflammatory cytokines, and in vivo therapeutic effects were assessed.

\section{Results}

We observed Nes-GFP+ cells colocalized with splenic stromal cells and further demonstrated that these Nes-GFP+ cells had the ability to establish ectopic lymphoid-like structures in vivo. Moreover, we showed that the Nes-GFP+ cells possessed the characteristics of MSCs. Spleen-derived Nes-GFP+ cells exhibited greater immunomodulatory ability in vitro, and more remarkable therapeutic efficacy in inflammatory diseases, especially inflammatory bowel disease (IBD) than bone marrow-derived Nes-GFP+ cells.

\section{Conclusions}

Overall, our data showed that Nes-GFP+ cells contributed to subsets of spleen stromal populations and possessed the biological characteristics of MSCs with a stronger immunoregulatory function and therapeutic potential than bone marrow-derived Nes-GFP+ cells.

\section{Introduction}

Mesenchymal stromal cells (MSCs) were originally considered nonhematopoietic stem cells isolated from bone marrow that participate in the formation of the bone marrow hematopoietic microenvironment and play an obvious supporting role in the proliferation and differentiation of hematopoietic stem cells[1]. They can self-renew, differentiate into osteoblasts, adipocytes and chondrocytes, and have a fibroblastlike morphology with notable plastic-adherent properties. In recent years, scientists have explored the intrinsic physiological roles that MSCs play in vivo and identified nutritional support and immune 
regulation functions, which highlight their therapeutic potential in tissue injury repair and inflammatory disease. Exogenous MSC-related clinical applications involve graft versus host disease[2], osteoarthritis[3], rheumatoid arthritis[4], lupus[5], myocardial infarct[6], stroke[7], and inflammatory bowel disease[8]. Although many preclinical experiments choose bone marrow-derived MSCs for cell-based therapies, limited evidence has proven that MSCs from different sources differ in tissue repair and immunomodulatory ability, suggesting that we might look for better sources for specific diseases.

Large numbers of studies have proven that the main mechanisms of MSC therapy are regulation of the development, differentiation and function of B and T lymphocytes[9]. The spleen is the main site for the development of $T$ and $B$ lymphocytes in adult tissues[10]. Stromal cell subpopulations such as follicular dendritic cells (FDCs) and marginal reticular cells (MRCs) in the B lymphocyte region and fibroblast reticular cells (FRCs) in the T lymphocyte region play an important role in the survival, differentiation and maturation of lymphocytes in the spleen[11]. Previous studies have found that FRCs, MRCs and FDCs express several cell surface markers similar to MSCs, such as CD73, CD90, CD105, CD157, PDGFRa, and Sca-1[12], suggesting that stromal cells in the spleen may have a functional correlation with MSCs. Karin Tarte et al. found that bone marrow-derived MSCs showed phenotypes of FRCs under the stimulation of TNF- $\alpha$ and lymphotoxin- $\alpha 1 \beta 2[13]$. Tonsil-derived MSCs exhibit the characteristics of FDCs stimulated by cytokines and TLR3 agonists. Moreover, FRCs have strong immunosuppressive ability, including inducing immune tolerance, inhibiting $T$ cell proliferation, and promoting Treg proliferation, to prevent the occurrence of autoimmune diseases, which is similar to the immunomodulatory function of MSCs[14]. Similarly, Fletcher al et al. showed that FRCs isolated from lymph nodes were far more effective than bone marrow-derived MSCs in the treatment of septic mice[15]. Therefore, we wanted to explore whether stromal cells residing in the spleen can be defined as spleen-derived MSCs and whether a complex immune environment might endow them with stronger immunomodulatory ability and better clinical application potential than bone marrow-derived MSCs. However, the lack of a common marker to identify MSCs from different tissues poses a limitation when comparing the general performance of different isolated populations. The phenotype and markers of mesenchymal stem/progenitor cells in adult spleens remain unknown.

Nestin, originally found in neuroepithelial stem cells, is an intermediate filament protein expressed in the early stages of development[16]. More importantly, Nestin is also expressed in some adult stem/progenitor cell populations, indicating that Nestin might be a common marker of multipotent stem cells. Using Nestin as a tracking marker, various adult stem cells have been prospectively isolated, including mesenchymal stem cells and hair follicle stem cells, suggesting that it can be used as a specific marker for isolating tissue-resident MSCs[17-20].

Here, we report a method for the identification and isolation of stromal cells in the spleen on the basis of Nestin expression using flow cytometry in combination with in vitro functional assays. Our assays provided evidence that these cell lines exhibited MSC characteristics and further demonstrated that Nestin-positive stromal cells in the spleen had the capacity to partially restore lymph node construction in 
vivo following transplantation into the kidney capsule and possessed a greater immunoregulatory function than bone marrow-derived MSCs.

\section{Materials And Methods}

\section{Animals}

Homozygous Nestin (Nes)-GFP transgenic mice on the C57BL/6 genetic background were provided by Dr Masahiro Yamaguchi[33]. C57BL/6 wild-type mice were purchased from the Animal Center at the Medical Laboratory of Guangdong Province, China. Nestin-cre mice (Stock No: 003771) and Rosa26-tdTomato $\left(R 26 R^{t d T}\right)$ reporter mice (Stock No: 007905) were obtained from the Jackson Laboratory. All specific pathogen-free mice used for studies were housed and handled according to protocols approved by the Sun Yat-sen University Institutional Animal Care and Use Committee.

\section{Isolation and culture of Nes-GFP+ cells from Nes-GFP transgenic mice}

Nes-GFP+ cells derived from mouse spleens were collected as previously described[18]. Briefly, the spleens were minced into small pieces with $5 \mathrm{~mL}$ of HBSS digestive solution including Type IV collagenase and incubated in a water bath at $37^{\circ} \mathrm{C}$ for $30 \mathrm{~min}$. After adding DMEM containing $10 \%$ fetal bovine serum (FBS, HyClone) to stop digestion, the cell suspensions were centrifuged at $1250 \mathrm{~g}$ for $5 \mathrm{~min}$ at room temperature, washed twice with phosphate-buffered saline (PBS) and filtered through a 40- $\mu \mathrm{m}$ cell strainer to prepare a single-cell suspension. Nes-GFP- cells and Nes-GFP+ cells were sorted using flow cytometry (Influx, BD) and cultured in DMEM/F12 (1:1) containing $1 \mathrm{ng} / \mathrm{mL}$ LIF (Millipore), $5 \mu \mathrm{g} / \mathrm{L}$ insulintransferrin-sodium selenite (ITS, Sigma-Aldrich), 5\% chicken embryo extract (US Biologicals), $0.1 \mathrm{mM} \beta$ mercaptoethanol (Invitrogen), 1\% nonessential amino acids (HyClone), 1\% N2 (Invitrogen), 2\% B27 (Invitrogen), $20 \mathrm{ng} / \mathrm{mL}$ bFGF (Invitrogen), and $20 \mathrm{ng} / \mathrm{mL}$ EGF (PeproTech). These cells were kept at $37^{\circ} \mathrm{C}$ in a humidified $5 \% \mathrm{CO} 2$ water-jacketed incubator and were propagated every 3 days. Nes-GFP+ cells from similar passages were used in all assays.

\section{RNA isolation and quantitative real-time and reverse transcription PCR}

Total RNA was extracted from spleen tissues using TRIzol reagent (Invitrogen), and $1 \mu \mathrm{g}$ of RNA was reverse transcribed using a RevertAid First Strand cDNA Synthesis Kit (Thermo Scientific). The generated cDNA was subjected to real-time PCR with SYBR Green reagent (Roche). The primer sequences are listed in the Supplementary information, Table $\mathrm{S} 1$.

\section{Clonal sphere formation assay}

Isolated single-cell suspensions of Nes-GFP+ cells were diluted to a density of 500 cells $/ \mathrm{mL}$, after which 2 $\mu \mathrm{L} /$ well of the diluted cell suspension was plated in ultralow attachment 96-well plates (Corning). Next, $150 \mu \mathrm{L}$ of expansion medium was added to each well. Wells containing only one cell were marked and 
observed daily. After 10 days in culture, we recorded single cells that had generated spheres $>50 \mu \mathrm{m}$ in diameter.

\section{Cell differentiation ability in vitro}

Osteogenic differentiation: Nes-GFP+ cells were cultured in a-MEM (Invitrogen) containing 20\% FBS (HyClone), $100 \mu \mathrm{g} / \mathrm{mL}$ ascorbic acid (Sigma-Aldrich), $100 \mathrm{nM}$ dexamethasone (Sigma-Aldrich), $10 \mathrm{mM} \beta-$ glycerophosphate (Sigma-Aldrich), and $100 \mathrm{lU} / \mathrm{mL}$ penicillin/streptomycin (Invitrogen) for 4 weeks. The medium was changed every 3 days. These osteogenic-differentiated cells were analyzed by staining with Alizarin Red to detect the presence of calcium.

Adipogenic differentiation: Nes-GFP+ cells were cultured in high-glucose DMEM containing $100 \mathrm{nM}$ dexamethasone (Sigma-Aldrich), $10 \mu \mathrm{g} / \mathrm{mL}$ insulin (Sigma-Aldrich), $0.2 \mathrm{mM}$ indomethacin (SigmaAldrich), $0.5 \mathrm{mM}$ 3-isobutyl-1-methylxanthine (Sigma-Aldrich), 10\% FBS (HyClone) and $100 \mathrm{IU} / \mathrm{mL}$ penicillin-streptomycin (Invitrogen) for 4 weeks. The medium was changed every 3 days. These adipogenic-differentiated cells were analyzed by staining with Oil red 0 .

Chondrogenic differentiation: Nes-GFP+ cells were cultured in a 15-mL conical tube with $2 \mathrm{~mL}$ of induction medium consisting of DMEM (Invitrogen) with $3 \%$ FBS (HyClone), $10 \mathrm{ng} / \mathrm{mL}$ tumor growth factor (TGF)- $\beta 3$ (PeproTech), $1 \times$ ITS (Sigma-Aldrich), and $1 \mathrm{mM}$ pyruvate (Sigma-Aldrich) for 4 weeks. The medium was changed every 3 days. Chondrocytes were identified by toluidine blue (Sigma-Aldrich) staining.

\section{Flow cytometric analysis}

Flow cytometric sorting was performed with an Influx apparatus (BD), while flow cytometric analyses were performed with Influx or Gallios (Beckman Coulter) flow cytometers. Data were analyzed with FlowJo 10.6.2 software (Treestar). The primary and secondary antibodies are listed in the Supplementary information, Table $\mathrm{S} 2$.

\section{Immunofluorescence staining}

For immunofluorescence (IF) staining, the mouse spleens and transplants were fixed in 4\% PFA and dehydrated with $30 \%$ sucrose. After fixation, the tissues were cut into $5-\mu \mathrm{m}$ sections. The tissue sections were incubated with 5\% normal serum and $0.1 \%$ Triton X-100 (HyClone) in PBS for $1 \mathrm{~h}$ at room temperature, followed by incubation with primary antibodies at $4{ }^{\circ} \mathrm{C}$ overnight. The sections were then incubated with secondary antibodies at room temperature for $1 \mathrm{~h}$. Nuclei were counterstained with DAPI (4',6-diamidino-2-phenylindole) for $10 \mathrm{~min}$. The primary and secondary antibodies are listed in the Supplementary information, Table S3. Images were acquired using an LSM780 confocal microscope (Zeiss).

\section{Artificial lymphoid formation}


The protocol for the generation of artificial lymphoid-like structures was performed as follows: The isolated CD45-Nes-GFP- and CD45- Nes- GFP+ cells derived from mouse spleens were placed onto collagenous matrix (CS-35; Koken) and squeezed several times to allow cell adsorption into the scaffold. Scaffolds containing cells were kept on ice and transplanted under one kidney capsule of anesthetized C57BL/ 6 mice. After 3 weeks, we detected the lymphoid formation of these cells using specific antibodies against T cells (CD3) and B cells (B220).

\section{Cytokine assays}

T cells were cultured with or without Nes-GFP+ cells for 2 days. During the last $6 \mathrm{~h}$ of incubation, PMA (50 $\mathrm{ng} / \mathrm{mL}$ ), ionomycin $(500 \mathrm{ng} / \mathrm{mL})$, and brefeldin $A(B F A ; 10 \mu \mathrm{g} / \mathrm{mL}$ ) were added to the culture system (all from Sigma-Aldrich Aldrich). IFN-y and TNF-a were analyzed by flow cytometry.

\section{Inflammatory bowel disease (IBD) model}

IBD reactions were induce in mice with trinitrobenzenesulfonic acid (TNBS) (Sigma-Aldrich). On the day one, mice were covered with $150 \mu \mathrm{L}$ of a premixed solution containing TNBS. On the day seven, food was withdrawn from the mice for 24 hours, after which we delivered TNBS ( $2.5 \mathrm{mg}$ in $100 \mu \mathrm{L}$ of $50 \%$ ethanol) into the colon $4 \mathrm{~cm}$ proximal to the anus of mice anesthetized with pentobarbital. The control mice received $100 \mu \mathrm{L}$ of ethanol. One day after treatment, the mice were subjected to intravenous (i.v.) injection of one million cells.

\section{Contact hypersensitivity model}

CHS reactions were induced in mice with 2,4-dinitro-1-fluorobenzene (DNFB) as previously described[34]. Briefly, DNFB (Sigma-Aldrich, 0.5\% dissolved in 4:1 acetone/olive oil) was applied to the shaved back of each mouse (sensitization). After 5 days, the mice were challenged by epicutaneous application of $0.2 \%$ DNFB to the right ear. The control group consisted of mice challenged with $0.2 \%$ DNFB on the right ear without prior sensitization. Ear thickness was measured at 24, 48, 72, and $96 \mathrm{~h}$ post challenge by individuals blinded to the treatment status. The degree of swelling was calculated as the thickness of the right ear (challenged ear) minus the baseline thickness of the left ear (unchallenged ear). To compare the immunosuppressive effects of Nes-GFP+ cells on the CHS response, the mice were subjected to intravenous (i.v.) injection of one million cells on day 2 post challenge.

\section{Statistical analysis}

All data are presented as the mean \pm SD obtained from at least three independent experiments. Comparisons between the groups were performed using one-way analysis of variance (ANOVA). Analysis was performed using SPSS (version 22). A $P$ value less than 0.05 was considered statistically significant.

\section{Results}

\section{Characterization of Nestin expression in the spleen}


To identify the expression of Nestin in the mouse spleen, we assessed the GFP signal in the Nes-GFP transgenic mouse model expressing GFP driven by the Nestin (Nes) promoter using confocal microscopy. Similar to findings in the testis, kidney and heart $[18,19,21]$, we also observed GFP expression in the mouse spleen (Figure 1A). Additionally, we confirmed the expression of Nestin in the mouse spleen using Nes-Cre mice bred with Rosa26-td-Tomato $\left(R 26 R^{t d T}\right)$ reporter mice (Nes-Cre, $R 26 R^{t d T}$ ) (Figure S1A). To systematically evaluate the change in Nestin expression during postnatal development, we performed quantitative RT-PCR (qRT-PCR) to detect the mRNA expression levels of Nestin in the whole mouse spleen at postnatal days $7,14,28$, and 90 . Quantitative analysis showed a significant peak in Nestin expression on postnatal day 14 , which gradually decreased with age (Figure 1B).

To further characterize Nes-GFP+ cells and their correlation with stromal cells in the spleen, we examined the expression of several typical splenic stromal cell lineage markers, ER-TR7, CD35, and MadCAM-1, representing fibroblastic reticular cells (FRCs), follicular dendritic cells (FDCs), and marginal reticular cells (MRCs), respectively[22-24]. The results of immunofluorescence staining showed that Nestin colocalized with ER-TR7, CD35, and MadCAM-1 in the spleen (Figure 1C-E). Analysis of the staining patterns revealed that about $80 \%$ of Nes-GFP+ cells expressed markers of FRCs, FDCs, and MRCs (Figure 1F). We also detected the expression of other regular stromal cell markers: ICAM1, VCAM1, and CD31. Similarly, Nestin colocalized with ICAM1 and VCAM1, which are markers of lymphoid tissue organizer (LTo) cells[25] (Figure S1B), instead of CD31 (Figure S1C). Taken together, these results indicate that Nes-GFP+ cells in the mouse spleen may be an important subpopulation of nonendothelial spleen stromal cells.

\section{Isolation and characterization of Nes-GFP+ cells from the spleen}

We sorted cells from the spleens of Nes-GFP transgenic mice using flow cytometric sorting and found that Nes-GFP+ cells constituted about $0.55 \%$ of the total spleen cell population (Figure 2A-B). As Nestin has been identified as a potential marker for MSCs in various tissues[18, 19, 21, 26], we wondered whether Nes-GFP+ cells in the spleen could be splenic MSCs. To confirm this hypothesis, we first carried out a single-cell sphere formation assay in which single-cell suspensions derived from primary Nes-GFP+ cells were seeded by serial dilution into 96-well plates to demonstrate the self-renewal capacity of the Nes-GFP+ cells. After a 10-day culture in vitro, the isolated spleen-derived Nes-GFP+ cells yielded many clonal spheres demonstrating a strong proliferative capacity in vitro (Figure 2C). Second, we cultured these cells under defined differentiation conditions to characterize the trilineage differentiation capacity. We observed that the Nes-GFP+ cells could differentiate into osteocytes, adipocytes, and chondrocytes, as identified by Alizarin red, Oil Red O, and toluidine blue staining, respectively (Figure 2D). Third, we used flow cytometry to analyze some MSC-related surface markers in Nes-GFP+ cells. As expected, these cells were positive for CD29, CD90, CD106 and PDPN, but almost negative for CD11C, CD31 and CD 45 (Figure 2E). Given the MSC-like stem cell features of Nes-GFP+ cells isolated from spleens, we proposed that Nestin may be a potential marker of spleen-derived MSCs.

\section{Spleen-derived Nes-GFP+ cells could form secondary lymphoid organs}


Our results suggested that Nes-GFP+ cells could be the stem/progenitor cells of lymphoid stromal cells in the spleen, so we hypothesized that spleen-derived Nes-GFP+ cells have the potential to remain the stromal network of lymphoid organs. To investigate this hypothesis, we performed artificial lymphoid formation[27] to detect the ability of Nes-GFP+ cells to form intact lymphoid tissue. After isolating CD45Nes-GFP- and CD45-Nes-GFP+ cells from Nes-GFP transgenic mice, we transplanted these cells with collagen scaffolds into the kidney capsule of C57BL/ 6 wild-type mice for three weeks, and then stained the separated transplants with antibodies against T cell marker (CD3) and B cell marker (B220). The results of immunofluorescence staining showed a clear $\mathrm{T}$ cell zone and $\mathrm{B}$ cell zone in the transplants formed by CD45-Nestin+ cells (Figure 3A). Compared with the transplant formed by spleen-derived NesGFP- cells, the transplant formed by spleen-derived Nes-GFP+ cells had a similar structure with lymph node, which suggested that spleen-derived Nes-GFP+ cells could form intact lymphoid tissue-like organoids (Figure 3B-D). Taken together, our results indicate that Nes-GFP+ cells derived from the spleen have the ability to rebuild the lymphatic stromal cell network.

\section{Immunosuppressive properties of Nes-GFP+ cells derived from the spleen}

Bone marrow-derived MSCs are known to suppress the function of T cells. Our results showed that spleen-derived Nes-GFP+ cells exhibit similar properties to MSCs, so we hypothesized that spleen-derived Nes-GFP+ cells could also have immunosuppressive properties. To investigate the immunoregulatory abilities of Nes-GFP+ cells, we detected their effects on the proinflammatory cytokine production of CD3+ $T$ cells. After coculture with CD3+ T cells for $48 \mathrm{~h}$, the flow cytometry results showed that spleen-derived Nes-GFP+ cells significantly reduced the secretion of TNF-a (Figure 4A-B) and IFN-Y (Figure 4C-D) by CD3+ $T$ cells dose-dependently, and could even remain the effects at a ratio of 1: 20. Moreover, compared with that of bone marrow-derived Nes-GFP+ cells, spleen-derived Nes-GFP+ cells showed an even stronger suppressive effect at the same ratio (Figure 4E). Overall, the results revealed that spleen-derived NesGFP+ cells exhibit stronger immunosuppressive properties than bone marrow-derived Nes-GFP+ cells in vitro.

\section{Spleen-derived Nes-GFP+ cells as a potential source for the treatment of inflammatory diseases}

MSCs have shown significant therapeutic potential in many clinical trials of inflammatory diseases, so we postulated that spleen-derived Nes-GFP+ cells could also attenuate inflammation in vivo. To evaluate the treatment prospects of spleen-derived Nes-GFP+ cells, we compared the therapeutic potential of bone marrow-derived and spleen-derived Nes-GFP+ cells in inflammatory bowel disease (IBD). Our results showed that both of them alleviated TNBS-induced colitis by injection intravenously, but spleen-derived Nes-GFP+ cells exhibited a better therapeutic efficacy (Figure 5A-F). Specifically, the group treated by spleen-derived Nes-GFP+ cells showed reduced body weight loss and lower DAI scores (Figure 5A-B). Additionally, the average colon length was reduced to a lesser degree in the spleen-derived Nes-GFP+ cells group than bone marrow-derived Nes-GFP+ cells group (Figure 5C-D). Moreover, histopathologic analysis indicated that epithelium loss and inflammatory cell infiltration were more significantly reduced after treated by spleen-derived Nes-GFP+ cells (Figure 5E-F). 
We also compared the effects of spleen-derived and bone marrow-derived Nes-GFP+ cells on contact hypersensitivity (CHS) mice. We injected spleen-derived and bone marrow-derived Nes-GFP+ cells separately into CHS mice and measured ear swelling and cellular infiltration (Figure S2A).

Histopathological examination revealed that ear swelling and cellular infiltration were improved after the injection of Nes-GFP+ cells (Figure S2B). Additionally, analysis of ear thickness after spleen-derived Nes$\mathrm{GFP}+$ cell injection showed a peak at $48 \mathrm{~h}$ and then a gradual decrease. A similar trend was observed in bone marrow-derived Nes-GFP+ cell-injected mice, but ear swelling was more severe than that in spleenderived Nes-GFP+ cell-injected mice (Figure S2C). Moreover, Nes-GFP+ cells derived from the spleen showed a better potential to reduce proinflammatory cytokine-producing CD8+ effector T cells in vivo (Figure S2D).

Taken together, our results suggest that Nes-GFP+ cells isolated from spleen have better immunomodulatory function than Nes-GFP+ cells isolated from bone marrow in vivo.

\section{Discussion}

In this study, we sought to examine whether Nestin can serve as a novel marker for the identification and isolation of spleen stromal cells. We isolated Nes-GFP+ cells from the spleens of Nes-GFP transgenic mice and found that these cells had characteristics consistent with splenic mesenchymal cell subset population precursors. Nes-GFP+ cells in the spleen not only proliferated continuously but also showed highly clonogenic potential, which suggests that Nes-positive stromal cells were capable of self-renewal. Moreover, the cells showed mesenchymal lineage differentiation potential. In addition, Nes-GFP+ stromal cells derived from the spleen showed greater immunomodulation ability than bone marrow-derived NesGFP+ cells, as evidenced by the suppression of the proliferation and functions of $T$ lymphocytes in vitro and the improvement observed upon intravenous injection into IBD and CHS mice.

The intermediate filament protein Nestin is a widely employed marker of multipotent neural stem cells (NSCs), and we have previously shown that Nestin is important for the survival and self-renewal of NSCs during embryonic development[28]. In adults, Nestin expression is frequently detected in areas of regeneration, such as neural progenitors, mesenchymal stem cells, hair follicle stem cells and myogenic cells, where Nestin-positive cells might function as a reservoir of stem/progenitor cells capable of proliferation and differentiation. Therefore, we proposed Nestin as a candidate marker for splenic stromal cells, including FRCs, FDCs and MRCs. Moreover, Nes-GFP+ cells derived from the spleen also encompass a subset of stromal progenitor cells with lymphoid tissue organizer activity capable of supporting ectopic lymphoid-like structures under the kidney capsule. Previous studies by Castagnaro and colleagues confirmed that Nkx2-5+/Islet1+ mesenchymal precursors generate distinct spleen stromal cell subsets[29]. Nike Julia Krautler et al. also proved that FDCs arise from ubiquitous perivascular precursors expressing PDGFR- $\beta[30]$. Based on our work, we identified Nestin as a potential marker of a distinct subset of splenic stromal cells, and Nes-positive cells might act as splenic stromal progenitor cells. 
As the largest peripheral lymphoid organ, splenic stromal cells orchestrate highly structured microenvironments that maximize the efficacy of immune responses to pathogens. In the T cell area, FRCs associate with clusters of T cells and support the formation of a conduit network that facilitates the directed migration of hematopoietic cells[31]. In the B cell area, FDCs capture and present antigens to B cells and support the process of affinity maturation; in the marginal zone area, marginal reticular cells provide structural support and participate in conduit formation[24, 32]. Based on a complex immune milieu, it was deduced that splenic stromal cells might be endowed with a higher efficacy in immunoregulation than mesenchymal stromal cells from other tissue sources. Here, we demonstrate that when expanded ex vivo and cocultured with T cells, spleen Nes+MSCs showed greater immunocompetence than bone marrow Nes+MSCs in the control of T cell inflammatory factor secretion. When used in mouse inflammatory diseases therapy, such as IBD and CHS, spleen Nes+MSCs significantly controlled the inflammatory response, and the therapeutic effect was better than bone marrow Nes+MSCs.

\section{Conclusions}

In conclusion, we identified Nestin as a potential marker of splenic stromal cells, and splenic Nes-GFP+ cells exhibit the characteristics of mesenchymal stromal cells. Moreover, transplanted splenic Nes-GFP+ cells under the kidney capsule appeared to organize the formation of lymphoid compartments, which reminded us of LTo cells. Additionally, an in vivo IBD and CHS disease model and in vitro coculture experiments demonstrated that Nes-GFP+ cells derived from the spleen were excellent in immunomodulation. Thus, our findings provide new insight into an optimal source of mesenchymal stromal cells for cell-based therapy in overwhelming inflammatory and autoimmune diseases and inspire the generation of artificial secondary lymphoid organs.

\section{Declarations}

\section{Ethics approval and consent to participate}

All procedures performed in studies involving animals were conducted strictly following the Sun Yat-sen University Institutional Animal Care and Use Committee.

\section{Consent for publication}

Not applicable.

\section{Availability of data and materials}

All relevant data and materials are available from the authors upon reasonable request.

\section{Competing interests}

The authors have declared that no competing interests exist. 


\section{Funding}

This study was supported by the National Key Research and Development Program of China, Stem Cell and Translational Research (2018YFA0107203, and 2019YFA0110300), National Natural Science Foundation of China (32130046, 81730005, 31771616, 81900075, 82170540, and 81970109), Natural Science Foundation of Guangdong Province (2018A0303130305 and 2021A1515011759), Key Research and Development Program of Guangdong Province (2019B020234001, 2019B020236002, and 2019B020235002), Key Scientific and Technological Program of Guangzhou City (201803040011), Pioneering talents project of Guangzhou Development Zone (2017-L163), Pearl River S\&T Nova Program of Guangzhou (201906010095) and Key Research and Development Program of Guangdong Province(2019B020234001 and 2019B020236002).

\section{Authors' contributions}

Conceptualization, A.P.X. and X.R.Z.; Methodology, M.R.L., J.H. and R.H.D., W.Q.L., M.H.J.; Investigation, X.R.Z. and J.H.; Writing - Original Draft, J.H. and M.R.L.; Writing - Review \& Editing, A.P.X. and X.R.Z.; Funding Acquisition, W.Q.L., M.H.J., A.P.X., and X.R.Z. Resources, R.H.D.; Supervision, A.P.X.

\section{Acknowledgements}

The authors gratefully acknowledge the contribution of Pr Andy Peng Xiang for the very helpful discussion and the careful review of the manuscript.

\section{References}

1. Benedetto Sacchetti, A.F., Stefano Michienzi, Silvia Di Cesare, Stefania Piersanti, Isabella Saggio, Enrico Tagliafico, Stefano Ferrari, Pamela Gehron Robey, Mara Riminucci, Paolo Bianco, SelfRenewing Osteoprogenitors in Bone Marrow Sinusoids Can Organize a Hematopoietic Microenvironment. cell, 2007. 131(2): p. 324-336.

2. Le Blanc, K., et al., Mesenchymal stem cells for treatment of steroid-resistant, severe, acute graftversus-host disease: a phase II study. The Lancet, 2008. 371(9624): p. 1579-1586.

3. Zhang, S., et al., MSC exosomes mediate cartilage repair by enhancing proliferation, attenuating apoptosis and modulating immune reactivity. Biomaterials, 2018. 156: p. 16-27.

4. Shin, T.H., et al., Human umbilical cord blood-stem cells direct macrophage polarization and block inflammasome activation to alleviate rheumatoid arthritis. Cell Death Dis, 2016. 7(12): p. e2524.

5. Wang, D., et al., Allogeneic mesenchymal stem cell transplantation in severe and refractory systemic lupus erythematosus: 4 years of experience. Cell Transplant, 2013. 22(12): p. 2267-77.

6. Duran, J.M., et al., Bone-derived stem cells repair the heart after myocardial infarction through transdifferentiation and paracrine signaling mechanisms. Circ Res, 2013. 113(5): p. 539-52.

7. Bang, O.Y., et al., Autologous mesenchymal stem cell transplantation in stroke patients. Ann Neurol, 2005. 57(6): p. 874-82. 
8. Panés, J., et al., Expanded allogeneic adipose-derived mesenchymal stem cells (Cx601) for complex perianal fistulas in Crohn's disease: a phase 3 randomised, double-blind controlled trial. The Lancet, 2016. 388(10051): p. 1281-1290.

9. Shi, Y., et al., Immunoregulatory mechanisms of mesenchymal stem and stromal cells in inflammatory diseases. Nat Rev Nephrol, 2018. 14(8): p. 493-507.

10. Mebius, R.E. and G. Kraal, Structure and function of the spleen. Nat Rev Immunol, 2005. 5(8): p. 60616.

11. den Haan, J.M., R.E. Mebius, and G. Kraal, Stromal cells of the mouse spleen. Front Immunol, 2012. 3: p. 201.

12. Cheng, H.W., et al., Origin and differentiation trajectories of fibroblastic reticular cells in the splenic white pulp. Nat Commun, 2019. 10(1): p. 1739.

13. Ame-Thomas, P., et al., Human mesenchymal stem cells isolated from bone marrow and lymphoid organs support tumor B-cell growth: role of stromal cells in follicular lymphoma pathogenesis. Blood, 2007. 109(2): p. 693-702.

14. Krishnamurty, A.T. and S.J. Turley, Lymph node stromal cells: cartographers of the immune system. Nat Immunol, 2020. 21(4): p. 369-380.

15. Fletcher AL, E.J., Astarita J, Murray R, Saeidi N, D'Rozario J, Knoblich K, Brown FD, Schildberg FA, Nieves JM, Heng TS, Boyd RL, Turley SJ, Parekkadan B., Lymph node fibroblastic reticular cell transplants show robust therapeutic efficacy in high-mortality murine sepsis. Sci Transl Med., 2014. 13(6): p. 249 ra109.

16. Lendahl U, Z.L., McKay RD., CNS Stem Cells Express a New Class of Intermediate Filament Protein. Cell, 1990. 60(4): p. 585-95.

17. Zulewski, H., et al., Multipotential Nestin-Positive Stem Cells Isolated From Adult Pancreatic Islets Differentiate Ex Vivo Into Pancreatic Endocrine, Exocrine, and Hepatic Phenotypes. Diabetes, 2001. 50(3): p. 521-533.

18. Jiang, M.H., et al., Characterization of Nestin-positive stem Leydig cells as a potential source for the treatment of testicular Leydig cell dysfunction. Cell Res, 2014. 24(12): p. 1466-85.

19. Liao, Y., et al., Cardiac Nestin(+) Mesenchymal Stromal Cells Enhance Healing of Ischemic Heart through Periostin-Mediated M2 Macrophage Polarization. Mol Ther, 2020. 28(3): p. 855-873.

20. Amoh, Y., et al., Multipotent nestin-positive, keratin-negative hair-follicle bulge stem cells can form neurons. Proceedings of the National Academy of Sciences of the United States of America, 2005. 102(15): p. 5530-5534.

21. Jiang, M.H., et al., Nestin(+) kidney resident mesenchymal stem cells for the treatment of acute kidney ischemia injury. Biomaterials, 2015. 50: p. 56-66.

22. Katakai, T., et al., A novel reticular stromal structure in lymph node cortex: an immuno-platform for interactions among dendritic cells, T cells and B cells. Int Immunol, 2004. 16(8): p. 1133-42. 
23. Fischer, M.B., et al., Dependence of Germinal Center B Cells on Expression of CD21/CD35 for Survival. Science, 1998. 280(5363): p. 582-585.

24. Katakai, T., et al., Organizer-like reticular stromal cell layer common to adult secondary lymphoid organs. J Immunol, 2008. 181(9): p. 6189-200.

25. Honda, K., et al., Molecular basis for hematopoietic/mesenchymal interaction during initiation of Peyer's patch organogenesis. J Exp Med, 2001. 193(5): p. 621-30.

26. Yin, Z., et al., Single-cell analysis reveals a nestin(+) tendon stem/progenitor cell population with strong tenogenic potentiality. Sci Adv, 2016. 2(11): p. e1600874.

27. Suematsu, S. and T. Watanabe, Generation of a synthetic lymphoid tissue-like organoid in mice. Nat Biotechnol, 2004. 22(12): p. 1539-45.

28. Li, W.Q., et al., Engraftable neural crest stem cells derived from cynomolgus monkey embryonic stem cells. Biomaterials, 2015. 39: p. 75-84.

29. Castagnaro, L., et al., Nkx2-5(+)islet1(+) mesenchymal precursors generate distinct spleen stromal cell subsets and participate in restoring stromal network integrity. Immunity, 2013. 38(4): p. 782-91.

30. Krautler, N.J., et al., Follicular dendritic cells emerge from ubiquitous perivascular precursors. Cell, 2012. 150(1): p. 194-206.

31. Bajenoff, M., N. Glaichenhaus, and R.N. Germain, Fibroblastic reticular cells guide T lymphocyte entry into and migration within the splenic T cell zone. J Immunol, 2008. 181(6): p. 3947-54.

32. Haberman, A.M. and M.J. Shlomchik, Reassessing the function of immune-complex retention by follicular dendritic cells. Nat Rev Immunol, 2003. 3(9): p. 757-64.

33. Yamaguchi, M., et al., Visualization of neurogenesis in the central nervous system using nestin promoter-GFP transgenic mice. NeuroReport, 2000. 11(9): p. 1991-1996.

34. Nagano, T., et al., Inhibitory effects of dietary soyasaponin on 2,4-dinitrofluorobenzene-induced contact hypersensitivity in mice. Exp Dermatol, 2017. 26(3): p. 249-254.

\section{Figures}


A

B
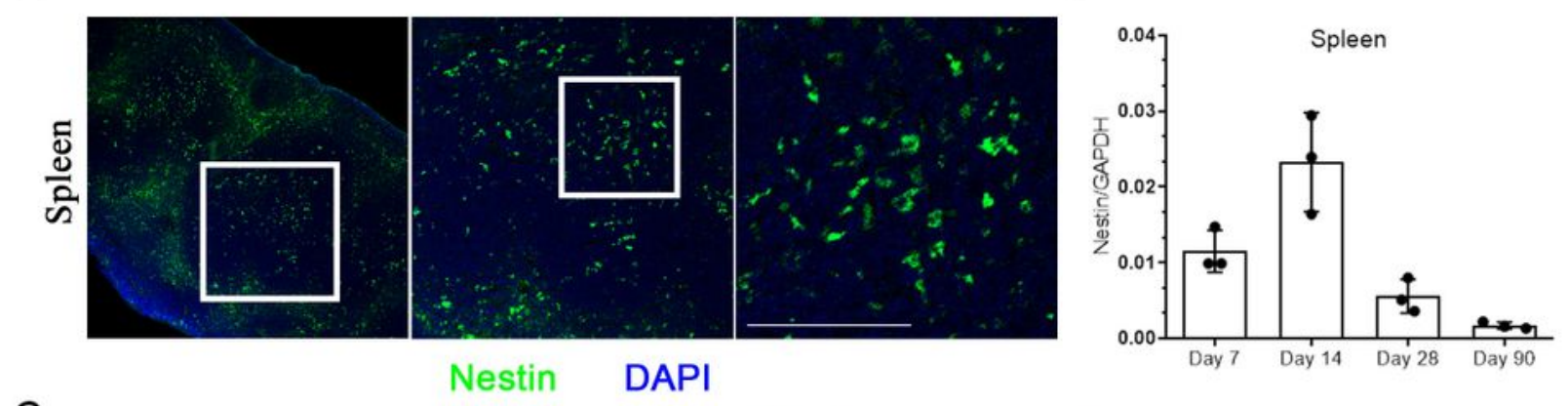

C
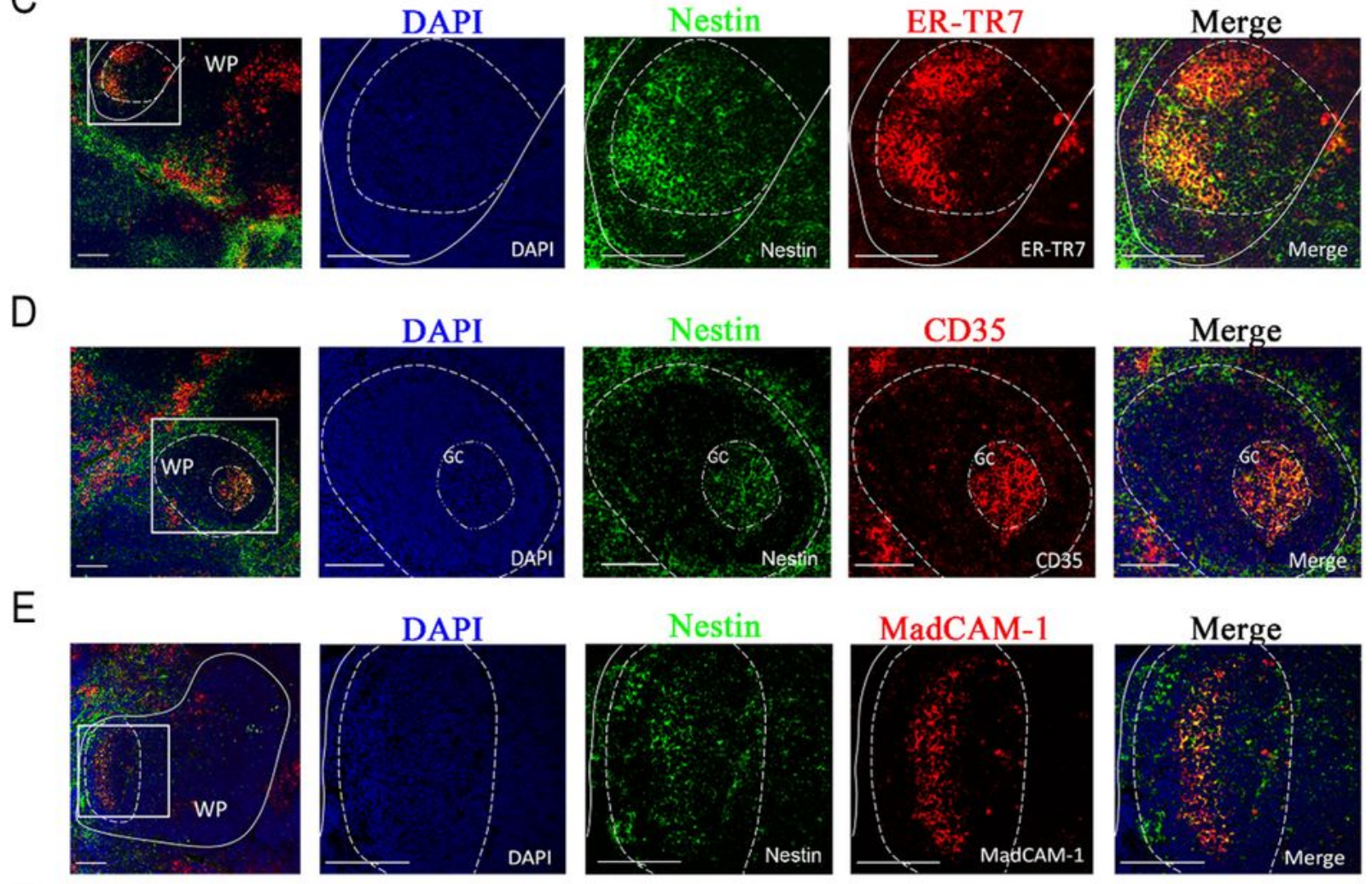

$\mathrm{F}$
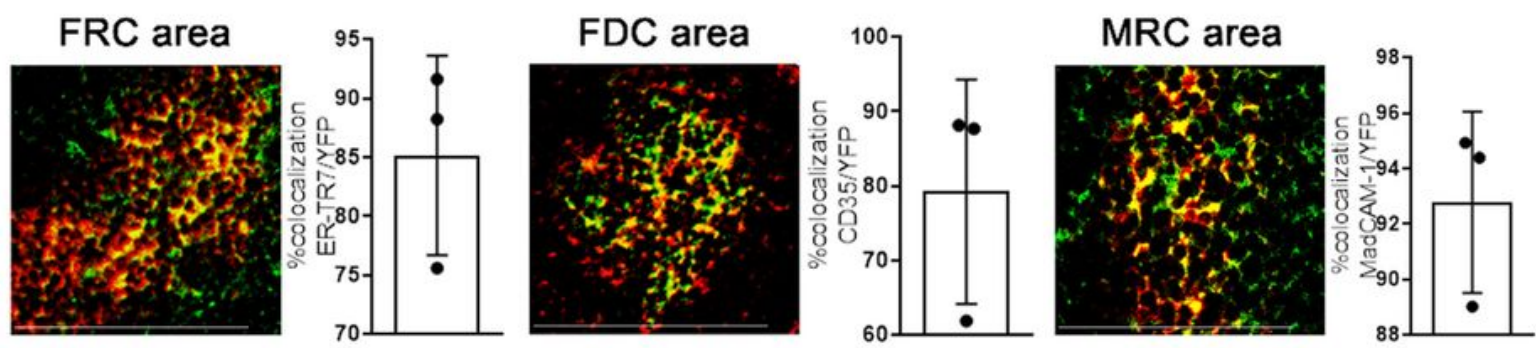

\section{Figure 1}

Characterization of Nestin expression in the spleen (A) Nestin (green) expression in the spleens of NesGFP transgenic mice. Nuclei were counterstained with DAPI (blue). Scale bar, $100 \mu \mathrm{m}$. (B) qRT-PCR analysis of Nestin gene expression in spleens of normal mice on postnatal days $7,14,28,90$. The relative mRNA expression levels of Nestin were normalized to reference GAPDH controls. $n=3$. (C-E) Immunohistochemical analyses showed that the Nes-GFP+ cells in the spleens expressed markers of 
FRCs, FDCs, and MRCs. (C) ER-TR7 (FRCs), (D) CD35 (FDCs), (E) MadCAM-1 (MRCs). Nuclei were counterstained with DAPI (blue). Scale bar, $50 \mu \mathrm{m}$. White Pulp, WP; Germinal Center, GC. (F) The percentage of Nes-GFP+ cells expressing ER-TR7, CD35, and MadCAM-1 was counted. $n=3$.

A

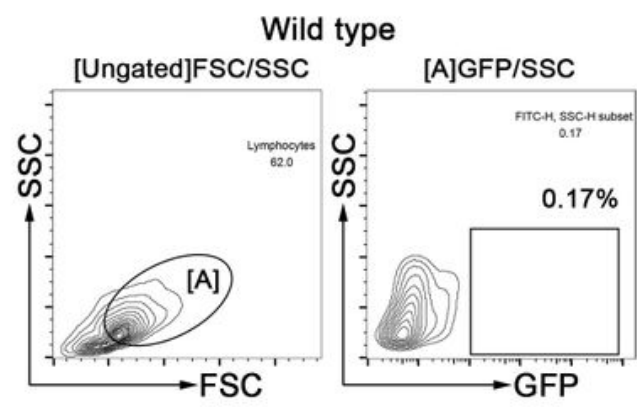

B

C
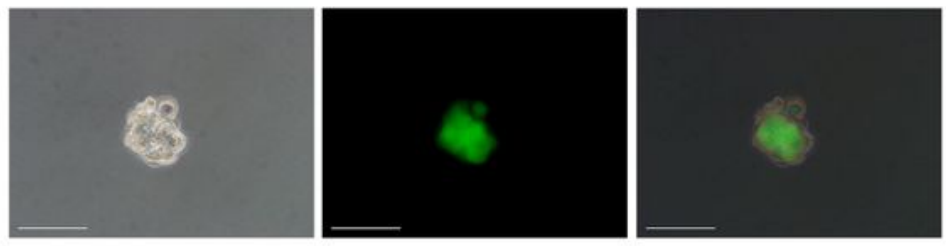

D

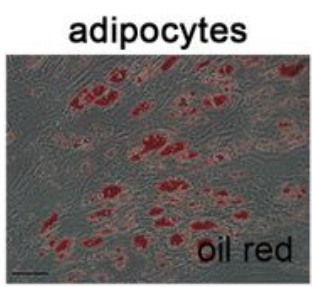

osteocytes

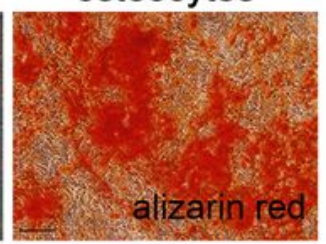

chondrocytes

E
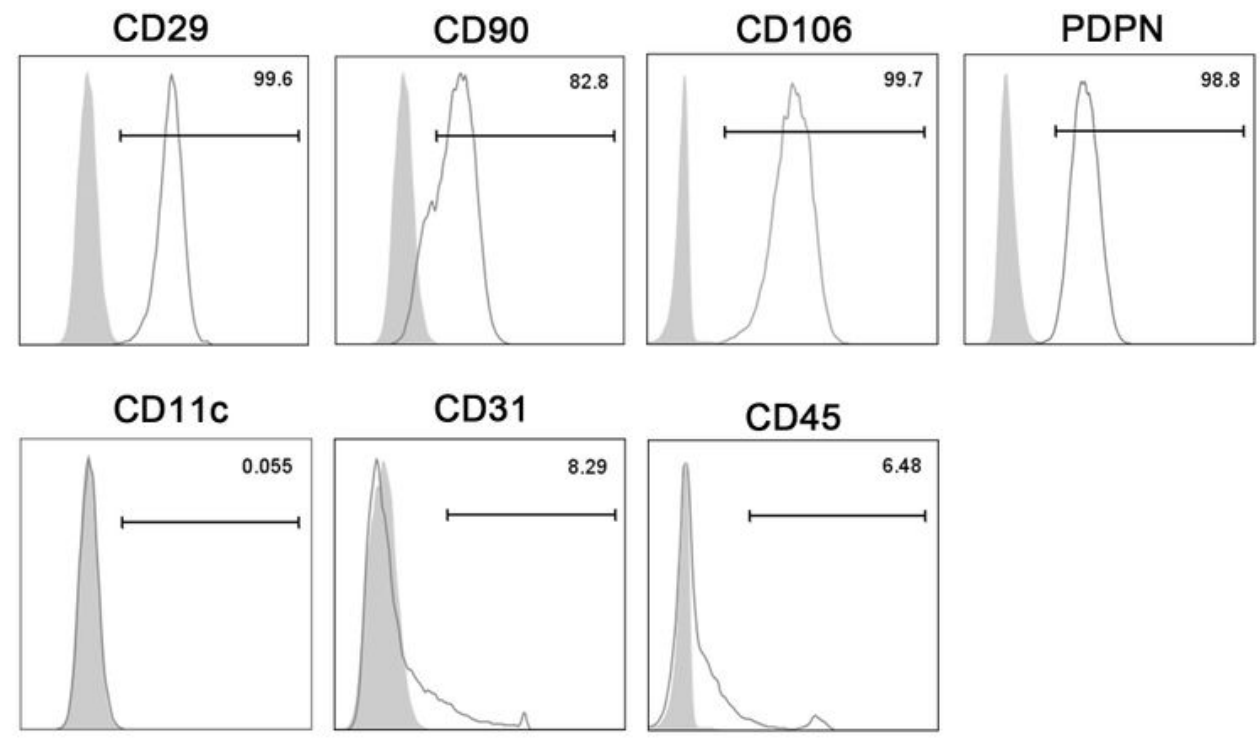

Figure 2

Isolation and characterization of Nes-GFP+ cells from the spleen (A, B) Flow cytometry was used to isolate Nes-GFP+ cells from the spleens of Nes-GFP transgenic mice. Cells from wild-type C57BL/ 6 mice 
were isolated as a control. (C) Representative images showing the clonal sphere growth of single NesGFP+ cells were observed using a bright field (left panel) or fluorescence (middle panel). Scale bars, 50 $\mu \mathrm{m}$. (D) Representative stained images showing that mouse spleen-derived Nes-GFP+ cells could differentiate into osteocytes (Alizarin red), adipocytes (Oil red O), and chondrocytes (toluidine blue). Scale bars, $100 \mu \mathrm{m}$. (E) The expression of cell surface markers on Nes-GFP+ cells was detected by flow cytometry.

A

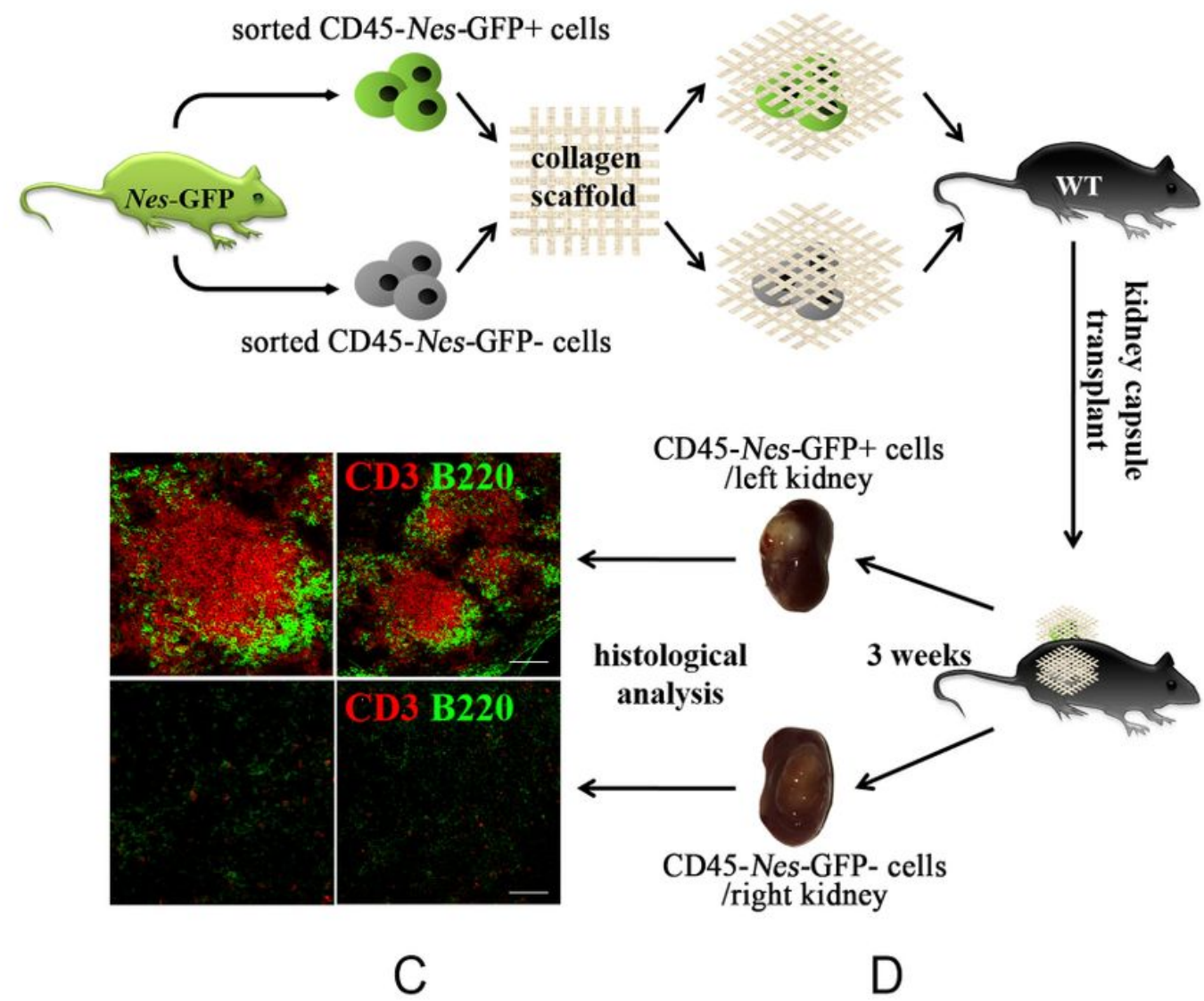

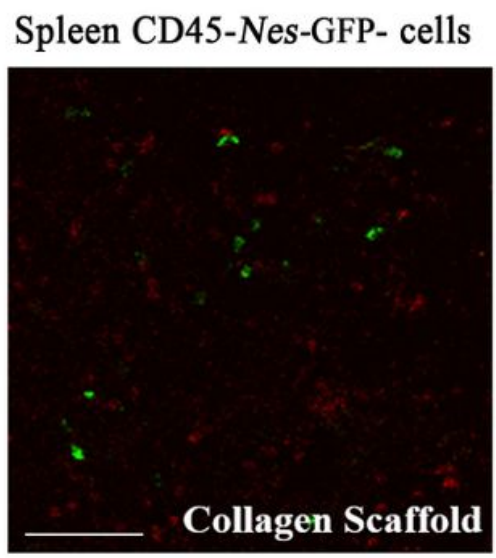

CD3 B220

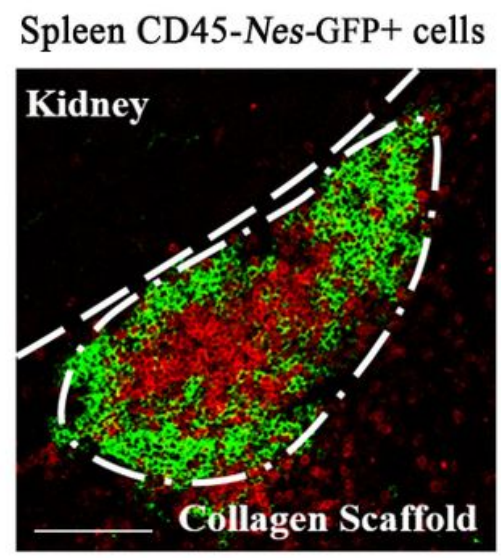

CD3 B220
Lymph node

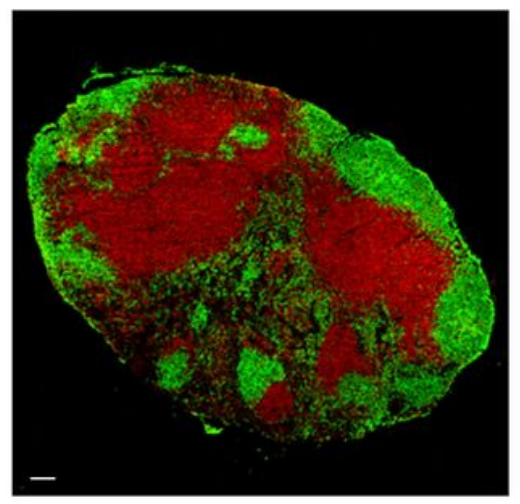

CD3 B220

\section{Figure 3}

Spleen-derived Nes-GFP+ cells could form secondary lymphoid organs (A) Spleen CD45-Nes-GFP- and CD45-Nes-GFP+ cells of Nes-GFP transgenic mice with collagen scaffolds were transplanted into the 
kidney capsule of wild-type mice. Scale bars, $100 \mu \mathrm{m}$. (B, C) Representative stained images of transplants showed that Nes-GFP+ cells, instead of Nes-GFP-cells, could form lymphoid tissue-like organoids. Scale bars, $100 \mu \mathrm{m}$. (D) Representative stained images of lymph node. Scale bars, $100 \mu \mathrm{m}$.

A
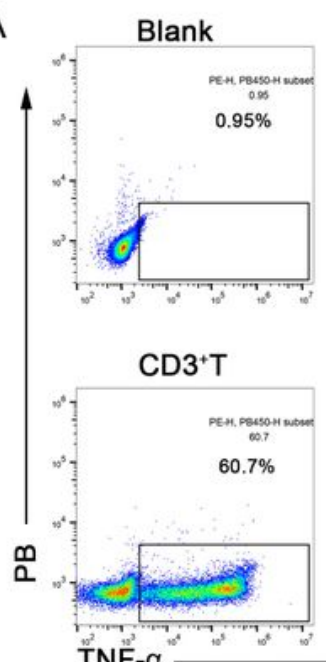

TNF- $\alpha$

C

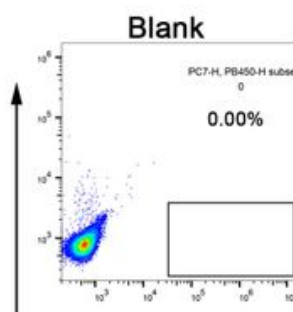

$\mathrm{CD}^{+} \mathrm{T}$

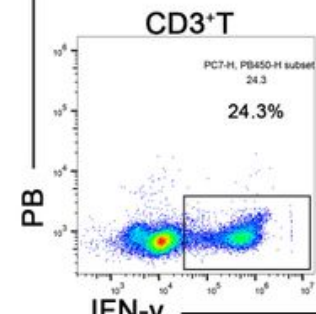

$E$

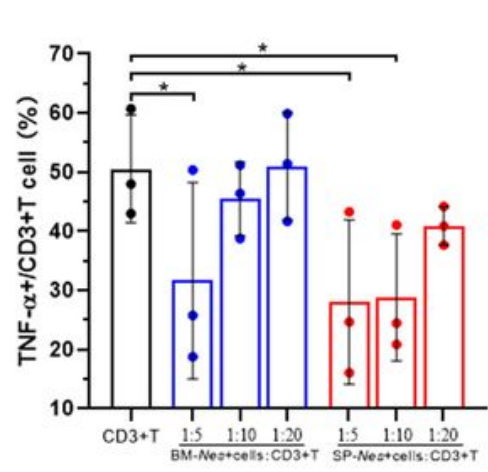

$\mathrm{CD}^{+} \mathrm{T}+\mathrm{BM}-\mathrm{Nes}+$ cells: $\mathrm{CD}^{+} \mathrm{T}$

B

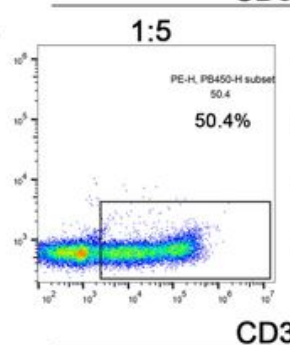

CD3
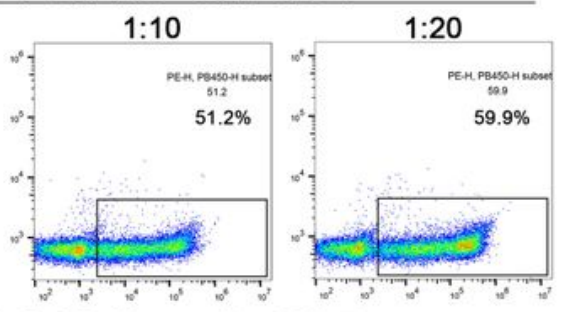

1:20

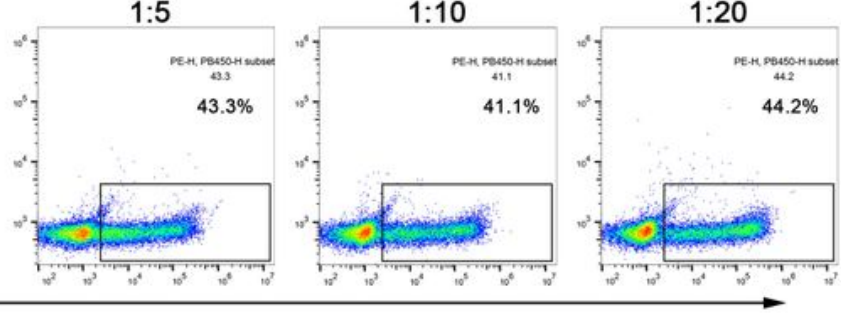

$\mathrm{CD}^{+} \mathrm{T}+\mathrm{BM}-\mathrm{Nes}+$ cells: $\mathrm{CD}^{+}{ }^{+} \mathrm{T}$

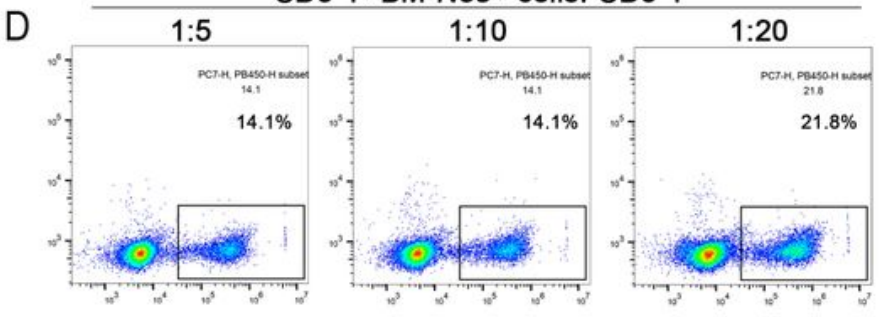

$\mathrm{CD}^{+} \mathrm{T}+\mathrm{SP}-\mathrm{Nes}+$ cells: $\mathrm{CD}^{+} \mathrm{T}$

$1: 5$

1:10

1:20
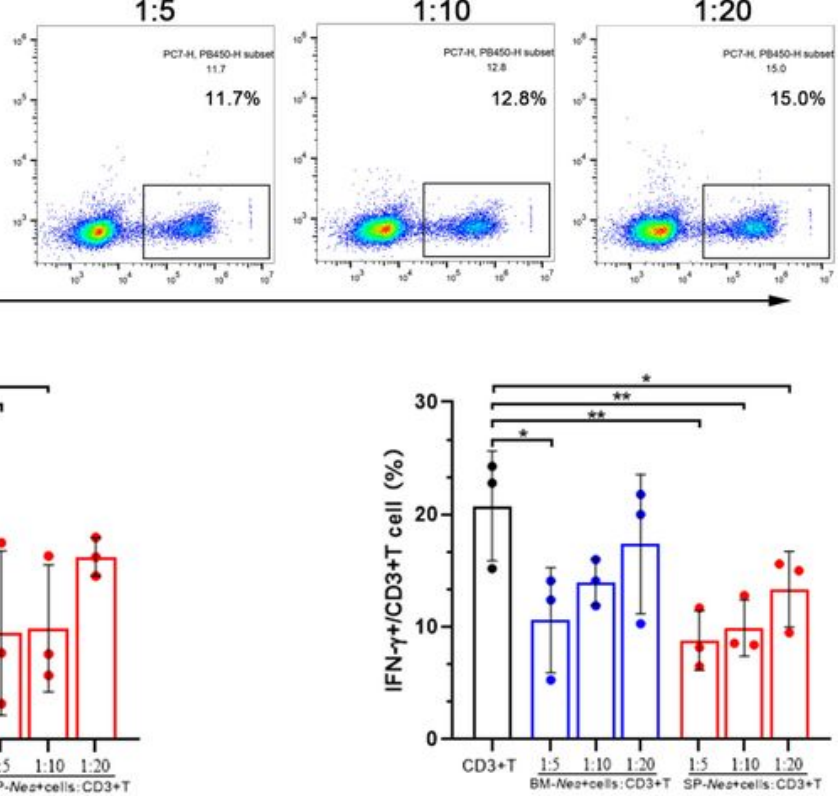

\section{Figure 4}

Immunosuppressive properties of Nes-GFP+ cells derived from the spleen (A, B) The production of IFN-y and TNF-a in cocultured T cells was examined by flow cytometry. The results are representative of three 
independent experiments. (C, D) The production of IFN- $\gamma$ and TNF- $\alpha$ in cocultured T cells was examined by flow cytometry. The results are representative of three independent experiments. (E) Bar graphs show the inhibition rate of IFN- $\gamma$ - and TNF-a-producing CD3+ T cells after coculture with Nes-GFP+ cells. ${ }^{*} \mathrm{P}<$ $0.05, * * \mathrm{P}<0.01, * * * \mathrm{P}<0.001$

A

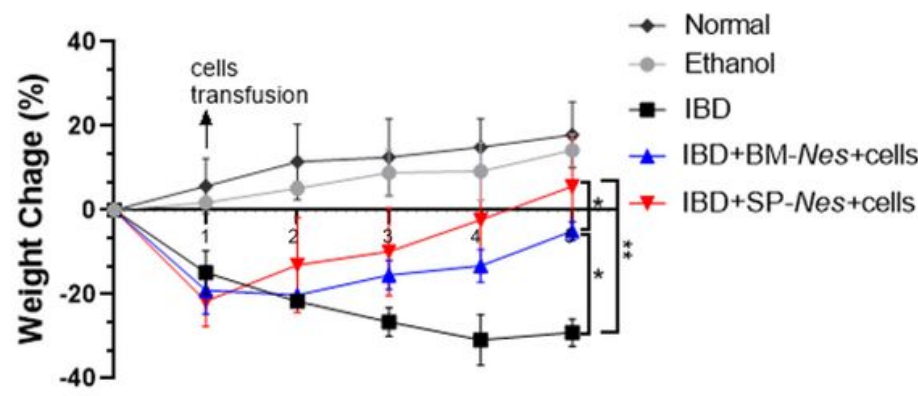

C

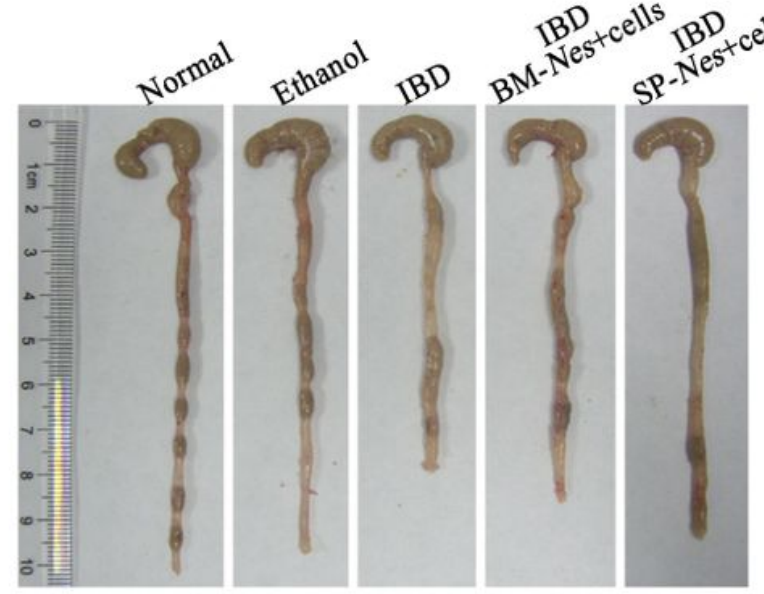

D
B

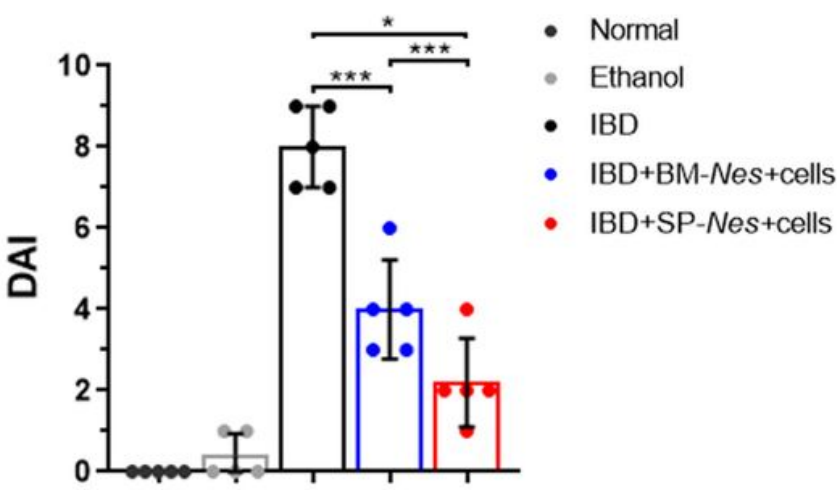

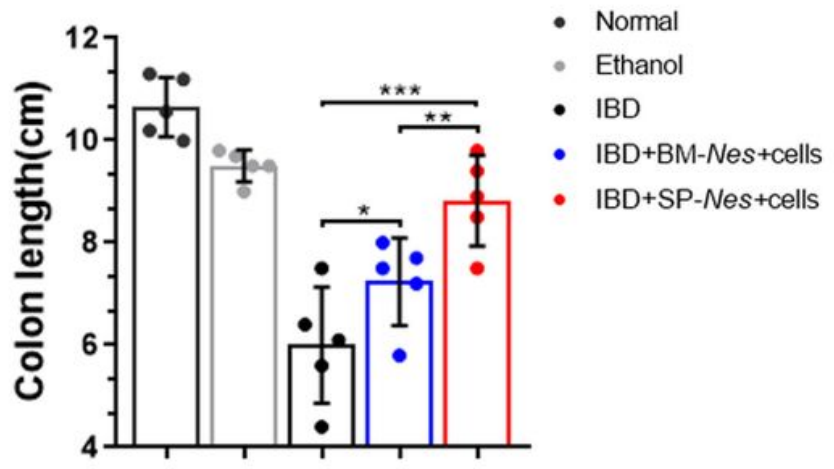

$E$
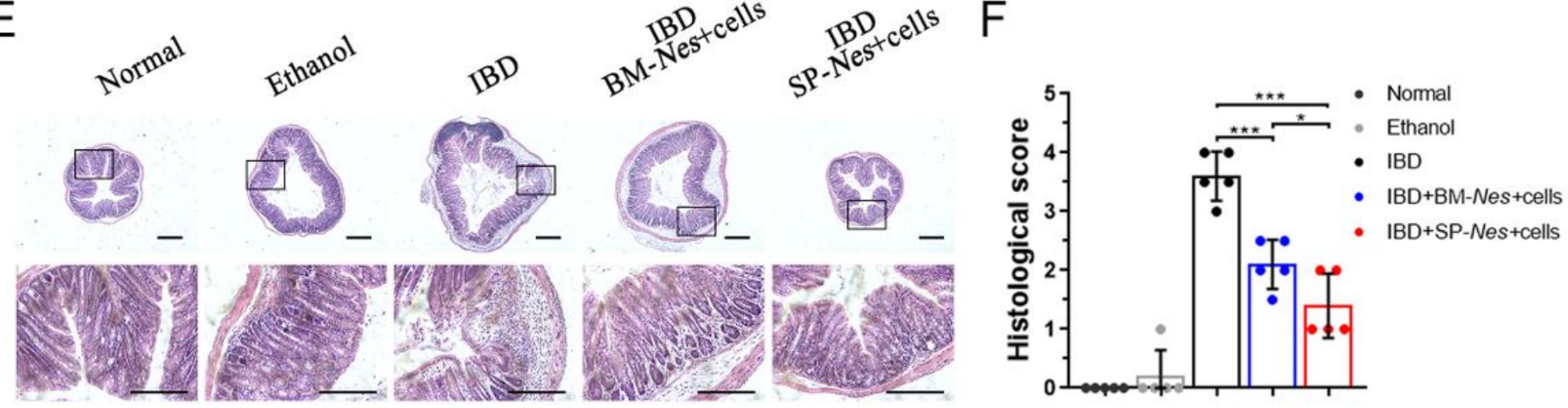

Figure 5

Spleen-derived Nes-GFP+ cells as a potential source for the treatment of inflammatory diseases (A) Therapeutic efficacy evaluation of TNBS-induced IBD model, bodyweight change percentage of each group. $n=3$. (B) Disease activity index (DAl) were measured. $n=5$. (C) Representative colonic length of mice. (D) Quantification of the colonic length of mice. $n=5$. $(E-F)$ H\&E staining and corresponding histological scoring of each group. $\mathrm{n}=5$. Scale bars, $100 \mu \mathrm{m}$. ${ }^{*} \mathrm{P}<0.05,{ }^{\star *} \mathrm{P}<0.01,{ }^{\star \star *} \mathrm{P}<0.001$ 


\section{Supplementary Files}

This is a list of supplementary files associated with this preprint. Click to download.

- supplementarymaterials.docx 